\title{
Displasia Cleidocraneal: Manejo ortodóncico y quirúrgico, seguimiento a 10 años.
}

\section{Cleidocranial Dysplasia: Orthodontic and surgical management, 10 year follow-up.}

\author{
1. Pontificia Universidad Católica de Chile. Chile \\ * Correspondencia autor. Carlos Izquierdo Aguirre \\ | Dirección: Salvador 95, oficina 210, Providencia, \\ Región Metropolitana | Teléfono: +569 87996848| \\ E-mail: carlosizq@gmail.com \\ Trabajo recibido el 21/02/2019. \\ Aprobado para su publicación el 17/03/2019
}

José Hassi ${ }^{1}$, Carlos Izquierdo ${ }^{1 *}$, Gonzalo Narea ${ }^{1}$

\begin{abstract}
RESUMEN
El tratamiento de trastornos óseos genéticos a nivel dentomaxilofacial suele ser muy complejo e invasivo. La displasia cleidocraneal es una condición que se caracteriza por generar a nivel oral hiperdoncia, y en consecuencia, apiñamiento dentario, retardo en la erupción y retención de dientes temporales, entre otros. En este caso el paciente presentaba dentición mixta primera fase a los 13 años de edad, fue tratado en conjunto con un equipo multidisciplinario donde se ha logrado un tratamiento mínimamente invasivo mediante cirugías por cuadrante y ortodoncia, consiguiendo alineamiento adecuado, nivelación y una oclusión lo más estable posible, además de una estética aceptable. El tratamiento expuesto demuestra que se pueden lograr buenos resultados con planificaciones conservadoras, sin embargo, existe una necesidad de alto compromiso y adhesión al tratamiento por parte del paciente y su familia.
\end{abstract}

PALABRAS CLAVE:

Displasia cleidocraneal, Ortodoncia, Cirugía oral, Tratamiento conservador.

Rev. Clin. Periodoncia Implantol. Rehabil. Oral Vol. 12(2); 109-112, 2019.

\section{ABSTRACT}

The treatment of genetic bone disorders at dentomaxillofacial level is usually very complex and invasive. Cleidocranial dysplasia is a condition that is characterized by oral hyperdontia, and consequently, dental crowding, delayed eruption and retention of temporary teeth, among others. In this case the patient had first phase mixed dentition at the age of 13 , he was treated collectively by a multidisciplinary team. A minimally invasive treatment was achieved through surgery and orthodontics, reaching proper alignment, leveling and an occlusion as stable as possible, in addition to acceptable aesthetics. The present approach shows that good results can be achieved with conservative planning. However, one of the keys for success is the patient's commitment.

KEY WORDS:

Cleidocranial dysplasia, Orthodontics, Oral surgery, Conservative treatment.

Rev. Clin. Periodoncia Implantol. Rehabil. Oral Vol. 12(2); 109-112, 2019

\section{INTRODUCCIÓN}

La Displasia Cleidocraneal (DCC) o disostosis cleidocraneal, es un raro trastorno óseo congénito con una herencia autosómica dominante de penetración completa, pero con expresión de fenotipo variable. Este fenotipo es caracterizado a nivel general, por aplasia o formación clavicular deficiente, imperfección y retraso de osificación de huesos del cráneo y talla baja, entre otras anomalías esqueléticas. A nivel estomatológico, encontramos exfoliación retardada en dientes primarios, impactación de dientes, y múltiples supernumerarios ${ }^{(1)}$.

\section{Etiología Genética}

Según las investigaciones genéticas realizadas se ha descubierto que la DCC suele ser causada por una haploinsuficiencia, fenómeno por el cual algunos genes expresan un fenotipo anormal cuando no se encuentran en la dosis génica adecuada. En este caso, la alteración se produce en el gen RUNX2 que ha sido mapeado en el cromosoma $6 p 21 . R U N X 2$ que es un factor de transcripción que a su vez es un regulador central de la formación ósea y, por lo tanto, tiene un papel importante en la diferenciación de los osteoblastos y en la maduración de los condrocitos ${ }^{(2,3)}$.

\section{Características clínicas}

Las principales características clínicas de la DCC son hipoplasia o definitivamente aplasia de la clavícula, lo cual genera disminución de la movilidad de los hombros, que a menudo se aproximan anteriormente. Hay imperfección o retraso de osificación de las fontanelas que genera una configuración voluminosa del cráneo o una depresión de la línea media de la zona frontal. Estos pacientes se caracterizan además, por poseer una mandíbula puntiaguda e hipertelorismo ${ }^{(4)}$.

En la cavidad oral encontramos presencia de hiperdoncia que conlleva a apiñamiento y mala alineación dentaria. El origen de la hiperdoncia se debe a una hiperactividad de la lámina dental fetal ${ }^{(4)}$. En algunos casos, luego de extracciones dentarias, pueden quedar remanentes de lámina dental que podrían dar origen a nuevos supernumerarios $^{(4)}$. Otras manifestaciones orales que se pueden 
encontrar son: erupción retardada y retención de piezas temporales y definitivas, anomalías anatómicas de las corona dentarias, hipoplasias de esmalte, quistes dentígeros y taurodontismo ${ }^{(5)}$.

\section{Diagnóstico diferencial}

Dentro de los diagnósticos diferenciales de la DCC se encuentran, entre otros, el síndrome de Crane-Heise, la displasia mandibuloacreal, la picnodisostosis, el síndrome de Yunis varon, La craneosinostosis-anomalías anales-poroqueratosis o síndrome de CDAGS y la hypophosphatasia. Estas condiciones, a diferencia de la DCC, son desórdenes autosómicos recesivos y pueden tener otras manifestaciones específicas, sin embargo, poseen características morfológicas comunes. Algunas de estas condiciones son causadas por mutaciones en genes que afectan la acción de RUNX2 sobre sus objetivos blancos ${ }^{(1)}$

La presencia de hipoplasia clavicular es fuertemente sugerente de DCC, pero esta anomalía incluso puede presentarse como una entidad aislada, sin formar parte de una unidad sindromática. En este caso, suele ser unilateral. La ausencia completa de ambas clavículas es una manifestación del Síndrome de Yunis-Varon (Online Mendelian Inheritance in Man, OMIM 216340). Esta última entidad igualmente se puede presentar con alteraciones cognitivas, anomalías en manos, pies y macizo facial y del cráneo, y malformaciones en otros sistemas, como el cardiovascular ${ }^{(1)}$.

Tanto en la DCC como en la picnodisostosis, se puede encontrar un defecto en la osificación craneal lo que condiciona la mantención de la fontanela anterior abierta y alteraciones de las suturas de los huesos Wormianos, alteración que también puede encontrarse en la osteogénesis imperfecta y en el hipotiroidismo congénito(1).

Otro elemento clínico importante para hacer el diagnóstico de DCC es la hiperdoncia. Se debe tener en consideración, sin embargo, que esta condición además se puede presentar en forma esporádica o familiar $^{(6)}$. También puede formar parte de un síndrome como el Síndrome de Gardner (OMIM 175100), caracterizado por poliposis familiar del colon, Síndrome de Hallerman-Streiff (OMIM 234100), en el cual se aprecia una cara angosta, hipotricosis y microftalmia, y el Síndrome orodigitofacial tipo I (OMIM 311200). Estas condiciones suelen presentarse con otras alteraciones que opacan el efecto de la hiperdoncia, por significar un mayor impacto en el desarrollo y la salud física general del paciente ${ }^{(1)}$.

\section{Manejo ortodoncia y cirugía}

Se han descrito múltiples enfoques para el manejo de la DCC, contrastantes y distintos entre sí en cuanto a la relación entre ortodoncia y cirugía.

El enfoque Toronto-Melbourne propone una serie de procedimientos quirúrgicos para extracción de piezas temporales dependiendo del desarrollo de las raíces de los permanentes, en una primera etapa a los 5-6 años y la segunda a los 9-10 años. En cada acto quirúrgico se eliminan además dientes supernumerarios que cubran a los permanentes correspondientes. Asimismo se considera exponer quirúrgicamente premolares y caninos para su posterior tracción ${ }^{(7)}$.

El enfoque Belfast-Hamburgo propone principalmente limitarse a un solo un acto quirúrgico mayor, donde se eliminen todos los dientes temporales y supernumerarios, y se expongan todos los permanentes. Bajo control ortodóncico se logra erupción de los permanentes ${ }^{(7)}$.

El enfoque Jerusalem se basa en una lógica que está relacionada directamente con el desarrollo dentoalveolar y los factores que causan anormalidad. El método tiene en cuenta cuatro aspectos principales que deben considerarse en su enfoque integral del tratamiento. Las características clínicas de las estructuras dentoalveolares, las medidas quirúrgicas para superarlas, la planificación de una estrategia de tratamiento ortodóncico para satisfacer las demandas inusuales de cada caso y concentrar los esfuerzos iniciales en la erupción de incisivos para lograr frente estético y mejorar imagen del paciente ${ }^{(7)}$.

Los aspectos clínicos de las estructuras dentoalveolares en mayor o menor grado como:

- Dientes temporales retenidos con raíces no reabsorbidas.

- Supernumerarios que modifican erupción de permanentes.

- Erupción retardada por pérdida de potencial eruptivo parcialmente.

- Disminución de tercio inferior, clase III esqueletal por hipodesarrollo de maxilar y rotación anterior de mandíbula.

- Tardía erupción de segundos molares definitivos.

- Retraso de alrededor de 3 años en desarrollo radicular dientes permanentes ${ }^{(7)}$.

Las medidas quirúrgicas con dos intervenciones en la edad dental de 7-8 años la primera y 10-11 años la segunda, donde principalmente: - Se extraen dientes temporales y supernumerarios
- Exponer dientes permanentes según la etapa de dentición mixta del paciente.

- Instalar aparatos inmediatamente.

- Cerrar los colgajos completamente(7).

Las condiciones que harán que la planificación ortodoncia sea adecuada son:

- Un número suficiente de dientes de anclaje erupcionados.

- El uso de un aparato de arco rígido.

- La aplicación de fuerzas suaves con un amplio rango de acción.

\section{CASO CLÍNICO}

Paciente género masculino de 23 años de edad, quien consultó a la edad de 13 años, derivado del Hospital Clínico de la Universidad de Chile a la Escuela de Odontología de la misma universidad por complejidad y oportunidad de tratamiento. El paciente fue diagnosticado a los 15 días de nacimiento con DCC, presenta talla baja e historia de cirugía de caderas. El examen clínico inicial y la exploración radiográfica reveló la presencia de características braquicefálicas y euriprosopas, paladar estrecho y ojival, dentición mixta primera fase, clase II y múltiples piezas supernumerarias y dientes permanentes retenidos sobre todo en maxilar superior.

El paciente no presentaba evidencias clínicas o de imágenes que demostraran alteraciones de otros sistemas como cardiovascular o genitourinario.

Dados los hallazgos mencionados, se planificó un tratamiento ortodóncico quirúrgico

El tratamiento se inició a los 13 años de edad, donde se planificó realizar, en primera instancia, exodoncia de dientes temporales del

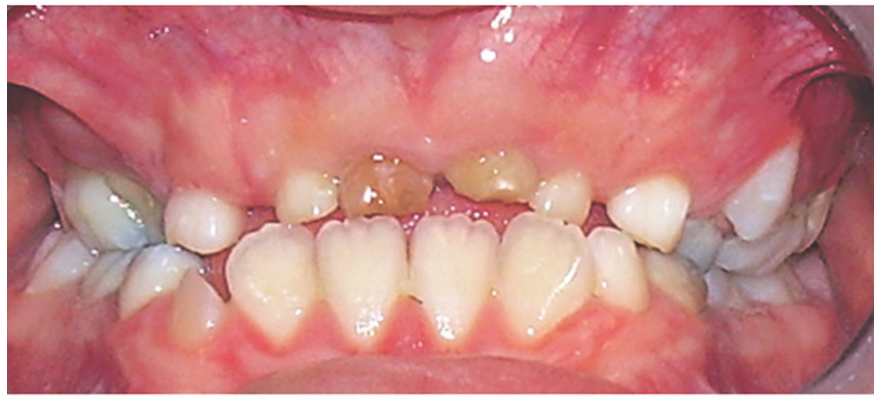

Figura 1. Estado inicial antes de tratamiento.

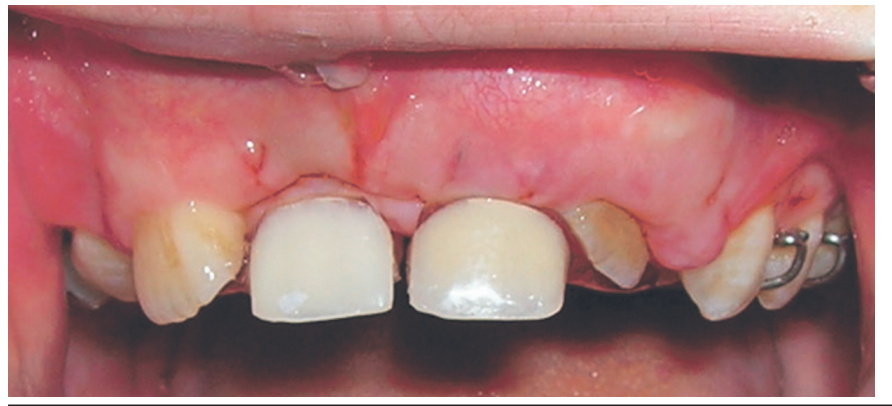

Figura 2. Estado luego de exodoncia de temporales e instalación de mantenedor de espacio estético.

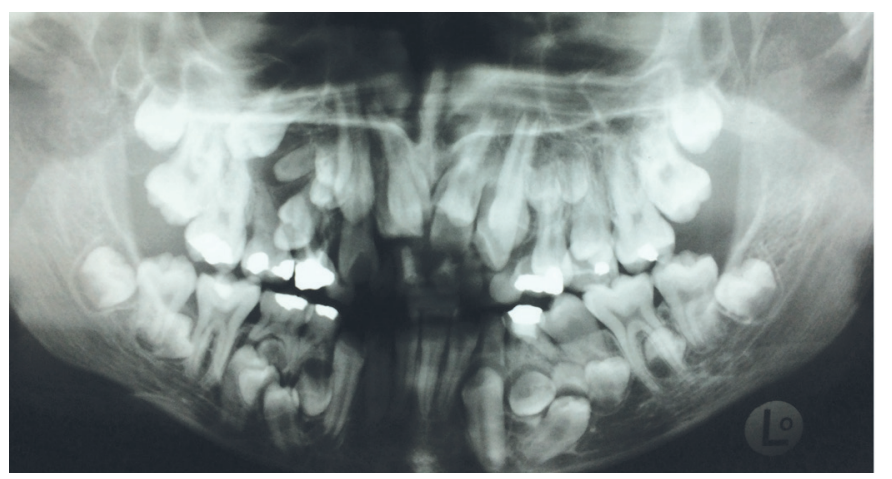

Figura 3. Radiografía panorámica inicial. 
grupo incisivo superior e instalar prótesis provisoria, para mejorar estética del paciente (Figura 1, 2 y 3).

A los 15 años de edad se decidió realizar la exodoncia de los 16 supernumerarios observados en los estudios radiográficos. Se realizó por cuadrantes en cuatro tiempos, separados por tres meses. (Figura 4 y 5$)$

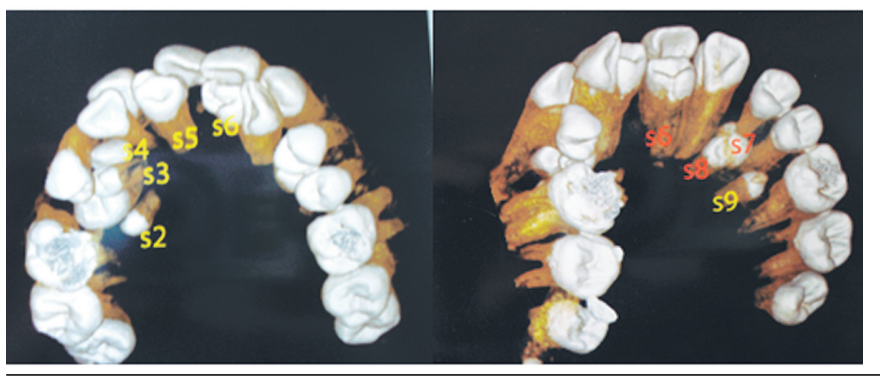

Figura 4. Conebeam CT superior para planificar cirugías.

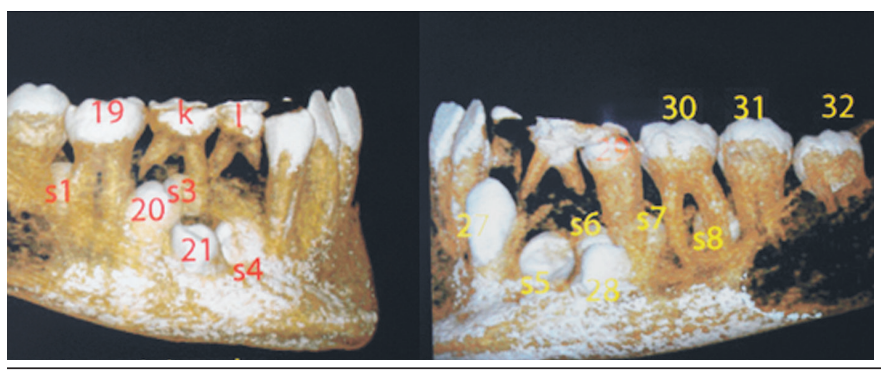

Figura 5. Conebeam CT inferior para planificar cirugías.

Luego de pausa de 3 años en que el paciente estuvo en control de crecimiento y desarrollo y evolución de dentición se decidió iniciar tratamiento ortodóncico. Se comenzó con el tratamiento de ortodoncia fijo con filosofía de McLaughlin, Bennett y Trivesi (MBT) principalmente por la versatilidad de la técnica, el uso de fuerzas ligeras continuas para aumentar el confort del paciente y minimizar la amenaza para el anclaje, el uso de una ranura de 0,022 " que permite una libertad de movimiento en los arcos iniciales y por tanto ayuda a limitar el nivel de fuerzas ${ }^{(8)}$. Se utilizó secuencia de arcos tradicional, comenzando con arcos 0,012" de niti circulares, avanzando hasta llegar a un arco 0,016 " por 0,016" de acero cuadrado en el presente.

Actualmente continúa en tratamiento, lográndose alinear la mayoría de las piezas dentarias y traccionar a su posición en el arco. Como objetivos de tratamiento actual se plantea:

- Fenestración y tracción al arco de pieza 4.5 y 3.4

- Exodoncia de supernumerarios restantes, los cuales no estaban formados al momento de las cirugías y están ubicados en relación a dientes a fenestrar.

- Mejorar paralelismo radicular.

- Mejorar estética mediante rehabilitación de los dientes transpocisionados y mediante periodoncia el alineamiento del margen gingival. (Figura 6 y 7 )

- Se estima que el tiempo necesario para terminar el tratamiento es alrededor de 12 a 18 meses.

\section{DISCUSIÓN}

La displasia cleidocraneal es un síndrome que genera alteraciones a nivel oral, es ahí donde el rol que el odontólogo juega es importante. Los problemas tanto a nivel estético como funcional que se generan en el paciente deben ser tratados por un equipo multidisciplinario incluyendo ortodoncista, cirujano, odontopediatra, rehabilitador y endodoncista si es que fuese necesario, dependiendo del caso.

Particularmente en este caso, a diferencia de los enfoques expuestos anteriormente, se optó por uno más conservador y menos invasivo, pero similar al enfoque de Jerusalem, realizando exodoncias en varios tiempos operatorios bajo anestesia local, en lugar de una cirugía mayor con anestesia general. Esta decisión fue tomada considerando las condiciones económicas y lugar de residencia del paciente, favoreciendo una atención efectiva y segura. Los mismos criterios se aplicaron para la planificación de los intervalos entre cirugías. El momento definido para comenzar con el tratamiento ortodóncico fue principalmente determinado por el retraso en el

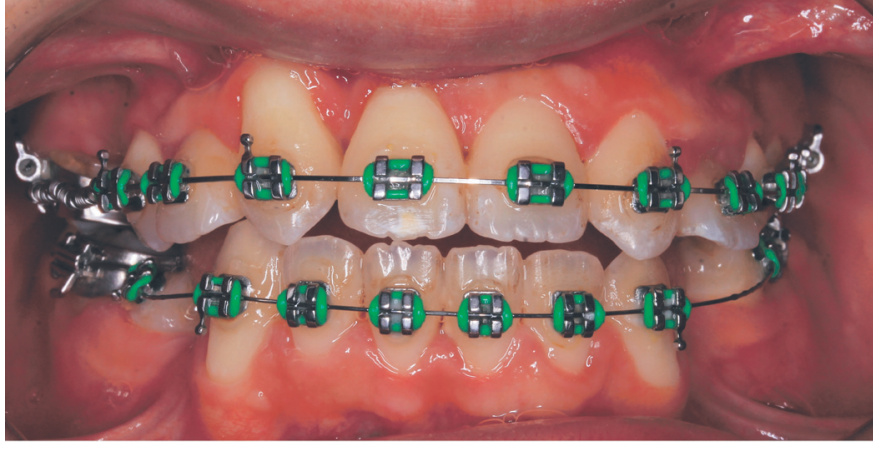

Figura 6. Estado actual de tratamiento.

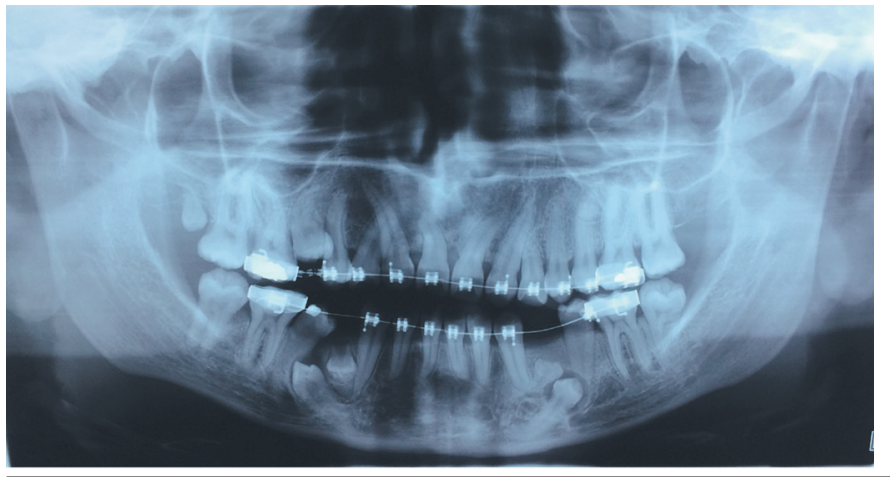

Figura 7. Radiografía panorámica actual.

crecimiento y desarrollo, además de la evolución dentaria. Esto ha sido reportado por Roberts et al(4).

La condición económica del paciente, así como la oportunidad de atención, hicieron que se priorizaran la solución y atención de otros problemas esqueletales y se postergara su condición dentocraneofacial, situación reportada también por Ferronato et $a^{(9)}$ y Rocha et al(10)

Otra condición especial reportada por Roberts et $\mathrm{al}^{(4)}$ que se dio en este paciente fue la aparición de nuevos supernumerarios, por desarrollo en forma tardía, luego de la exodoncia de los formados en una primera instancia, determinando una nueva intervención quirúrgica para el paciente.

Consideramos que para lograr un tratamiento exitoso son tres los puntos claves. En primer lugar, el diagnóstico temprano e inicio de tratamiento oportuno para poder realizar los procedimientos en el momento indicado. El segundo punto clave es la planificación multidisciplinaria del tratamiento. Por último, como en todo tratamiento ortodóncico, es fundamental el compromiso del paciente, ya que el tratamiento y tiempo de seguimiento puede involucrar un tiempo mucho mayor que otros más comunes de la especialidad.

Otro punto relevante, a modo de conclusión, es que al abordar estos casos es el tener presente que el proposito final no es lograr un resultado perfecto, sino lograr el mejor resultado posible, tanto estético como funcional, de acuerdo a las posibilidades del paciente y posibilidades del tratante. Es importante y debe ser considerado durante todo el tratamiento por el paciente, por el ortodoncista y el equipo de tratamiento.

\section{CONFLICTO DE INTERÉS}

Los autores declaran no tener conflicto de interés ni recibir financiamiento de ningún tipo.

\section{RELEVANCIA CLÍNICA}

El caso presentado a continuación representa un tratamiento multidisciplinario de complejidad mayor, llevado a cabo de manera conservadora con planificación en etapas de acuerdo a los distintos estadios de crecimiento y desarrollo del paciente, demostrando así la posibilidad de lograr con un adecuado seguimiento, tratamientos exitosos sin intervenciones mayores. 


\section{Bibliografía}

1. Toptancı I, Çolak H, Köseoğlu S. Cleidocranial dysplasia: Etiology, clinicoradiological presentation and management. J Clin Exp Invest. 2012; 3: 133-136.

2. Villegas Leon K. Caracterización de la expresión de Runx2 y Cbfß a través del ciclo celular, en células ROBmtert y MC3T3 [Memoria de título profesional]. Santiago, Universidad de Chile, 2008. Disponible en: http://repositorio.uchile.cl/ tesis/uchile/2008/villegas k/sources/villegas k.pdf act dic 2018.

3. Bufalino A, Paranaíba LMR, Gouvêa AF, Gueiros LA, Martelli-Júnior H, Junior JJ, et al. Cleidocranial dysplasia: oral features and genetic analysis of 11 patients. Oral Dis. 2012; 18, 184-190.

4. Roberts T, Stephen L, Beighton P. Cleidocranial dysplasia: a review of the dental, historical, and practical implications with an overview of the South African experience. Oral Surg Oral Med Oral Pathol Oral Radiol. 2013;115:46-55.

5. McNamara CM, O'Riordan BC, Blake M, Sandy JR. Cleidocranial dysplasia: radiological appearances on dental panoramic radiography. Dentomaxillofac Radiol. 1999;28:89-97.
6. Díaz A, Orozco J, Fonseca M. Multiple hyperodontia: report of a case with 17 supernumerary teeth with nonsyndromic association. Med Oral Patol Oral Cir Bucal. 2009;14:229-231.

7. Becker A, Lustmann J, Shteyer A. Cleidocranial dysplasia: Part 1-General principles of the orthodontic and surgical treatment modality. Am J Orthod Dentofac Orthop. 1997;111:28-33

8. McLAughlin R, Bennett J, Trevisi H. Breve historia y revisión de la mecánica de tratamiento. En: Costa M, Costa A, editor. Mecanica sistematizada del tratamiento ortodóncico. 2da edición. Madrid: Elsevier España SA; 2002. p 1-23.

9. Farronato G, Maspero C, Farronato D, Gioventù S. Orthodontic Treatment in a Patient with Cleidocranial Dysostosis. Angle Orthod. 2009; 79: 178-185.

10. Rocha R, Batistella Zasso M, Floriano G, Derech C, Ulema Ribeiro G, Locks A, et al. Orthodontic traction in a patient with cleidocranial dysplasia: 3 years of followup. Am J Orthod Dentofacial Ortho. 2014; 146: 108 - 118. 